\title{
Sums and products of intervals in ordered semigroups
}

\author{
T. Glavosits and Zs. Karácsony
}

\begin{abstract}
We show a simple example for ordered semigroup $\mathbb{S}=\mathbb{S}(+, \leqslant)$ that $\mathbb{S} \subseteq \mathbb{R}(\mathbb{R}$ denotes the real line $)$ and $] a, b[+] c, d[=] a+c, b+d[$ for all $a, b, c, d \in \mathbb{S}$ such that $a<b$ and $c<d$, but the intervals are no translation invariant, that is, the equation $c+] a, b[=] c+a, c+b[$ is not always fulfilled for all elements $a, b, c \in \mathbb{S}$ such that $a<b$.

The multiplicative version of the above example is shown too.

The product of open intervals in the ordered ring of all integers (denoted by $\mathbb{Z}$ ) is also investigated. Let $I_{x}:=\{1,2, \ldots, x\}$ for all $x \in \mathbb{Z}_{+}$ and defined the function $g: \mathbb{Z}_{+} \rightarrow \mathbb{Z}_{+}$by$$
g(x):=\max \left\{y \in \mathbb{Z}_{+} \mid I_{y} \subseteq I_{x} \cdot I_{x}\right\}
$$

for all $x \in \mathbb{Z}_{+}$. We give the function $g$ implicitly using the famous Theorem of Chebishev.

Finally, we formulate some questions concerning the above topics.
\end{abstract}

\section{Introduction}

It is well known from elementary real analysis [12], [2] that if $a, b, c, d$ are real numbers such that $a<b$ and $c<d$, then

$$
] a, b[+] c, d[=] a+c, b+d[,
$$

Key Words: interval, ordered dense Abelian group, ordered field

2010 Mathematics Subject Classification: Primary 39B22.

Received: 11.07 .2020

Accepted: 31.08 .2020 
moreover, if $0 \leqslant a<b$ and $0 \leqslant c<d$ then

$$
] a, b[\cdot] c, d[=] a c, b d[.
$$

We investigate the case when (1) or (2) remains valid in more general settings. Our references for ordered algebraic structure are [4], [9], [10], [11], [13].

Now we give a short list of necessary concepts:

We say that $X=X(\leqslant)$ is a partially ordered set or poset, if $X$ is a set and $\leqslant$ is a relation on $X$ such that it is reflexive, symmetric and transitive.

A poset $X=X(\leqslant)$ is said to be linearly ordered or loset, if $x \leqslant y$ or $y \leqslant x$ including the case $x=y$ for all $x, y \in X$.

We say, that the poset $X=X(\leqslant)$ is lattice ordered if every two elements of $X$ have a unique supremum (also called a least upper bound or join) and a unique infimum (also called a greatest lower bound or meet).

Let $X=X(*)$ be a groupoid in the sense that $X$ is a nonempty set and $*$ is a binary operation on $X$. Let $A, B$ be sets and $a \in X$. Define the sets and $A * B$ and $a * B$ by

$$
\begin{aligned}
A * B & :=\{a * b \in X \mid a \in A, b \in B\}, \\
a * B & :=\{a\} * B .
\end{aligned}
$$

Let $X=X(\leqslant)$ be a poset and $a, b \in X$ such that $a<b$, that is, $a \leqslant b$, but $a \neq b$, then the (open) interval with endpoints $a$ and $b$ is defined by

$$
] a, b[:=\{x \in X \mid a<x \text { and } x<b\} .
$$

An ordered semigroup $\mathbb{S}=\mathbb{S}(+, \leqslant)$ is a semigroup together with a partial ordering of its elements that is compatible with the group operations in the sense that if $x \leqslant y$, then $x+z \leqslant y+z$ for all $x, y, z \in \mathbb{S}$.

An ordered group $\mathbb{G}=\mathbb{G}(+, \leqslant)$ is a group together with a partial ordering of its elements that is compatible with the group operation.

Let $X=X(+, \leqslant)$ be an ordered semigroup (group). We say that the open intervals have translation invariant property if

$$
c+] a, b[=] c+a, c+b[
$$

for all $a, b, c \in \mathbb{S}$ such that $a<b$.

Let $\mathbb{S}=\mathbb{S}(\cdot, \leqslant)$ be an ordered semigroup. We say that the open intervals have homothety invariant property if

$$
c \cdot] a, b[=] c a, c b[
$$

for all $a, b, c \in X$ such that $a<b$. 
A poset $X$ is said to be dense if $] x, y[\neq \emptyset$ for all $x, y \in X$ with $x<y$.

Let $Y$ be a poset and $A \subseteq B \subseteq Y$. The set $A$ is said to be dense in the set $B$ if $] b_{1}, b_{2}\left[\cap A \neq \emptyset\right.$ for all $b_{1}, b_{2} \in B$ such that $b_{1}<b_{2}$.

Our paper is structured as follows:

In section 2 we give example for ordered semigroup $\mathbb{S}(+, \leqslant)$ where $\leqslant$ is a lattice order and the intervals of this semigroup are translation invariant and thus the equation (1) is fulfilled.

In section 3 we investigate the product of open intervals of ordered ring of integers denoted by $\mathbb{Z}$ using the famous number-theoretical theorem of Chebishev.

In section 4 among the others we give examples for semigroups $\mathbb{S}(+, \leqslant)$ that equation (1) is fulfilled without equation (3).

In section 5 we formulate conjectures and problems concerning structures which are similar to the structures presented in the previous chapter.

\section{Sums of intervals in ordered semigroup}

Definition 2.1. Let $\mathbb{S}=\mathbb{S}(+, \leqslant)$ be an ordered Abelian semigroup ( $\leqslant$ is a partial order). Consider the following properties:

1. $\mathbb{S}=\mathbb{S}(+)$ is cancellative in the sense that $x+z=y+z$ implies $x=y$ for all $x, y, z \in \mathbb{S}$.

2. If $x<y$ then there exists an element $z \in \mathbb{S}$ such that $y=x+z$ for all $x, y, z \in \mathbb{S}$.

3. $x \neq x+y$ for all $x, y \in \mathbb{S}$.

4. The strickly order $<$ is co-directed in the sense that for all $x, y \in \mathbb{S}$ there exists an element $z \in \mathbb{S}$ such that $z<x$ and $z<y$.

Now we show that the intervals of the ordered semigroup $\mathbb{S}$ with properties of Definitions 2.1 are translation invariant.

Theorem 2.2. Let $\mathbb{S}=\mathbb{S}(+, \leqslant)$ be an ordered semigroup with properties of Definitions 2.1. Let $\alpha, \beta, \gamma \in \mathbb{S}$ such that $\alpha<\beta$. Then

$$
\gamma+] \alpha, \beta[=] \gamma+\alpha, \gamma+\beta[\text { and } \gamma+] \alpha, \beta]=] \gamma+\alpha, \gamma+\beta]
$$

where $] x, y]:=\{z \in \mathbb{S} \mid x<z$ and $z \leqslant y\}$ for all $x, y \in \mathbb{S}$.

Proof. The proof is trivial. 
Theorem 2.3. Let $\mathbb{S}=\mathbb{S}(+, \leqslant)$ be an ordered semigroup with properties of Definition 2.1. Let $a, b, c, d \in \mathbb{S}$ such that $a<b$ and $c<d$. Then

$$
] a, b[+] c, d[=] a+c, b+d[.
$$

Proof. It is easy to see that $] a, b[+] c, d[\subseteq] a+c, b+d[$. One can easily obtain the converse inclusion by the Theorem 2.2 .

Example 2.4. Let $X:=\mathbb{Q}^{2}$. Then $X(+, \leqslant)$ is an ordered semigroup with the partial order $\leqslant$ and the operation + defined by

$$
\begin{aligned}
& \left(x_{1}, x_{2}\right) \leqslant\left(y_{1}, y_{2}\right): \Longleftrightarrow x_{1} \leqslant y_{1} \text { and } x_{2} \leqslant y_{2}, \\
& \left(x_{1}, x_{2}\right)+\left(y_{1}, y_{2}\right):=\left(x_{1}+y_{1}, x_{2}+y_{2}\right)
\end{aligned}
$$

for all $\left(x_{1}, x_{2}\right),\left(y_{1}, y_{2}\right) \in X$. Then the relation $\leqslant$ is lattice order and $X$ has properties of Definition 2.1.

\section{The products of open intervals in ordered group of integers}

It is well-known that the sum of nonempty open interval of the ring of all integers (denoted by $\mathbb{Z}$ ) is also an interval, but the endpoints of the sum are not equal to the sum of endpoints. This fact is used in our paper [7] when we investigate restricted Pexider additive functional equations in the cases when the additivity is satisfied on a rectangular or on the union of two rectangles of $\mathbb{Z}^{2}$.

It is also well-known that the product of nonempty open interval of the ordered ring $\mathbb{Z}$ is not always an open interval. For example let

$$
I_{x}:=\{1,2, \ldots, x\} \quad\left(x \in \mathbb{Z}_{+}\right) .
$$

Then $I_{3}$ is an open interval of $\mathbb{Z}$, but $I_{3} \cdot I_{3}=\{1,2,3,4, *, 6, *, *, 9\}$ is not.

Define the function $g: \mathbb{Z}_{+} \rightarrow \mathbb{Z}_{+}$by

$$
g(x):=\max \left\{y \in \mathbb{Z}_{+} \mid I_{y} \subseteq I_{x} \cdot I_{x}\right\} \quad\left(x \in \mathbb{Z}_{+}\right) .
$$

It is easy to see that for example $g(3)=4$. Now we give a table which contains the value of $x$ and $g(x)$ for some small integer $x$.

\begin{tabular}{|c|c|c|c|c|c|c|c|c|c|c|c|c|c|}
\hline$x$ & 1 & 2 & 3 & 4 & 5 & 6 & 7 & 8 & 9 & 10 & 11 & 12 & 13 \\
\hline$g(x)$ & 1 & 2 & 4 & 4 & 6 & 6 & 10 & 10 & 10 & 10 & 12 & 12 & 16 \\
\hline
\end{tabular}

The above table suggests the following Theorem. The proof of this Theorem is based on the the Bertrand's postulate which states that there exists a prime 
number in the interval $[n, 2 n]$ for all $n \in \mathbb{Z}_{+}$. This postulate was proved for the first time by P. L. Chebishev in 1850 and simplified later by P. Erdős in 1932 [3] due to M. El Bachraoui [1].

Theorem 3.1. The function $g$ has the following properties:

1. the function $g$ is increasing;

2. $g(x-1)<g(x)$ if and only if $x$ is prime;

3. $g\left(p_{n}\right)=p_{n+1}-1$ where $p_{1}, p_{2}, \ldots$ is the increasing sequence of all prime numbers.

Proof. Let us assume that there exist $u, v \in \mathbb{Z}_{+}$such that $p_{n} \leqslant v<u v<p_{n+1}$. Since $v<u v$, then $u<2$. By the Theorem of Chebyshev $v \leqslant p_{n+1} \leqslant 2 v<u v$ which is a contradiction. Thus we have that if $x \in \mathbb{Z}_{+}$such that $p_{n}<x<$ $p_{n+1}$, then there exist $u, v \in \mathbb{Z}_{+}$such that $u, v \leqslant p_{n}$ and $x=u v$.

\section{Additional examples}

In this section we only investigate linearly ordered semigroups. For this we give some notations.

Define the set $K_{1}$ by

$$
K_{1}:=\left\{a+b \sqrt{2} \mid a \in \mathbb{Q}_{+}, b \in \mathbb{Q}_{+}\right\} .
$$

Then $K_{1}=K_{1}(+, \leqslant)$ and $K_{1}=K_{1}(\cdot, \leqslant)$ are ordered semigroups where + , . and $\leqslant$ are the usual addition, multiplication and order in the real line.

Define the set $\mathbb{Q}(\sqrt{2})$ by

$$
\mathbb{Q}(\sqrt{2}):=\{a+b \sqrt{2} \mid a \in \mathbb{Q}, b \in \mathbb{Q}\} .
$$

Then $\mathbb{Q}(\sqrt{2})=\mathbb{Q}(\sqrt{2})(+, \cdot, \leqslant)$ is an ordered field where the operation,$+ \cdot$ and the order $\leqslant$ is the usual field operation and order in the real line $\mathbb{R}$.

Let $p_{1}, p_{2}, \ldots, p_{n}$ be pairwise different prim numbers. Define the set $X_{n}$ by:

$$
X_{n}:=\left\{x_{1}, x_{2}, \ldots x_{2^{n}}\right\}
$$

where $x_{1}=1, x_{2}=\sqrt{p_{1}}, \ldots, x_{n+1}=\sqrt{p_{n}}, x_{n+2}=\sqrt{p_{1} p_{2}}, \ldots, x_{2^{n}}=$ $\sqrt{p_{1} \ldots p_{n}}$. Then we can construct two sets from the set $X_{n}$ by:

$$
\begin{aligned}
& K_{n}:=\left\{\sum_{i=1}^{2^{n}} a_{i} x_{i} \mid a_{i} \in \mathbb{Q}_{+}, x_{i} \in X_{n} \quad\left(i=1, \ldots, 2^{n}\right)\right\}, \\
& \mathbb{Q}_{n}:=\left\{\sum_{i=1}^{2^{n}} a_{i} x_{i} \mid a_{i} \in \mathbb{Q}, x_{i} \in X_{n} \quad\left(i=1, \ldots, 2^{n}\right)\right\} .
\end{aligned}
$$


In both of the above cases,$+ \cdot$ and $\leqslant$ are the usual addition, multiplication and order in the real line respectively. It is easy to see that $K_{n}=K_{n}(+, \cdot, \leqslant)$ is an ordered dense semiring and $\mathbb{Q}_{n}=\mathbb{Q}_{n}(+, \cdot, \leqslant)$ is an ordered field, consequently all of equations (3), (1), (4) and (2) are fulfilled in $\mathbb{Q}_{n}[8]$. It is also easy to see that $\mathbb{Q}_{n}=\mathbb{Q}\left(\sqrt{p_{1}}, \ldots, \sqrt{p_{n}}\right)$ is an ordered field $\left(\mathbb{Q}\left(\sqrt{p_{1}}, \ldots, \sqrt{p_{n}}\right)\right.$ denotes the smallest subfield of the real line that contains both elements $\sqrt{p_{1}}, \ldots, \sqrt{p_{n}}$ and all elements of the field $\mathbb{Q}$ ) [5].

For numerical examples we use constants which are defined by:

$$
\begin{array}{ll}
\alpha:=0.5+0.5 \sqrt{2}=1.2071 \ldots, & \beta:=0.5+\sqrt{2}=1.9142 \ldots, \\
\gamma:=2+\sqrt{2}=3.4142 \ldots, & \delta:=1+2 \sqrt{2}=3.8284 \ldots
\end{array}
$$

Thus we obtain that $\alpha+\gamma=4.6213 \ldots, \beta+\delta=5.7426 \ldots, \alpha \cdot \gamma=4.1213 \ldots$, $\beta \cdot \delta=7.3284 \ldots$

Proposition 4.1. Let $a, b, c, d \in K_{1}$ such that $a<b$ and $c<d$. Then

$$
] a, b[+] c, d[=] a+c, b+d[.
$$

Proof. The inclusion $] a, b[+] c, d[\subseteq] a+c, b+d[$ is clear. For the converse inclusion let

$$
\left.x_{0}:=u+v \sqrt{2} \in\right] a+c, b+d[.
$$

We find elements $x^{*}, y^{*} \in K_{1}$ such that

$$
\left.x^{*}=x+y \sqrt{2} \in\right] a, b\left[, \quad y^{*}=x_{0}-x^{*} \in\right] c, d[.
$$

Now we list the necessary and sufficient conditions for $\left.x^{*} \in\right] a, b\left[\right.$ and $y^{*} \in$ ]$c, d[$ :

1. $a<x^{*}$ which is equivalent to the inequality $e_{1}(x)<y$ where

$$
e_{1}(x)=-\frac{1}{\sqrt{2}} x+A_{1} \quad \text { and } \quad A_{1}=\frac{a}{\sqrt{2}}
$$

2. $x^{*}<b$ which is equivalent to the inequality $y<e_{2}(x)$ where

$$
e_{2}(x)=-\frac{1}{\sqrt{2}} x+A_{2} \quad \text { and } \quad A_{2}=\frac{b}{\sqrt{2}}
$$

3. $c<y^{*}$ which is equivalent to the inequality $y<e_{3}(x)$ where

$$
e_{3}(x)=-\frac{1}{\sqrt{2}} x+A_{3} \quad \text { and } \quad A_{3}=\frac{x_{0}-c}{\sqrt{2}}
$$


4. $y^{*}<d$ which is equivalent to the inequality $e_{4}(x)<y$ where

$$
e_{4}(x)=-\frac{1}{\sqrt{2}} x+A_{4} \quad \text { and } \quad A_{4}=\frac{x_{0}-d}{\sqrt{2}} .
$$

Now we list the necessary and sufficient conditions of $x^{*} \in K_{1}$ and $y^{*} \in K_{1}$ :

(5) $u-x>0$ which is equivalent to the inequality $x<u$;

(6) $v-y>0$ which is equivalent to the inequality $y<v$.

Define the domains $D_{1}$ and $D_{2}$ by

$$
\begin{aligned}
& D_{1}:=\left\{(x, y) \in \mathbb{Q}_{+}^{2} \mid e_{1}(x)<y, y<e_{2}(x), y<e_{3}(x), e_{4}(x)<y\right\}, \\
& D_{2}:=\left\{(x, y) \in \mathbb{Q}_{+}^{2} \mid x<u \text { and } y<v\right\} .
\end{aligned}
$$

$D_{1}$ is the set of all rational points of the first quadrant which points are contained by two strips. These strips are determined by the pairs of parallel lines $e_{1}, e_{2}$ and $e_{4}, e_{3}$ respectively.

The intersection of these two strips is not empty if and only if $] A_{1}, A_{2}[\cap$ ]$A_{4}, A_{3}\left[\neq \emptyset\right.$ (as open intervals of the real line) which is equal to $A_{1}<A_{3}$ and $A_{4}<A_{2}$. These last two inequalities are fulfilled.

Since $e_{1}(u)<v, e_{4}(u)=v$ and $\mathbb{Q}^{2}$ is dense in $\mathbb{R}^{2}$ thus $D_{1} \cap D_{2} \neq \emptyset$. Chose a point $P=P(x, y) \in D_{1} \cap D_{2}$ arbitrarily, and define the numbers $x^{*}, y^{*}$ by

$$
x^{*}:=x+y \sqrt{2} \quad \text { and } \quad y^{*}:=(u-x)+(v-y) \sqrt{2} .
$$

Then $\left.x^{*} \in\right] a, b\left[\right.$ and $\left.y^{*} \in\right] c, d[$ whence we obtain that $] a+c, b+d[\subseteq] a, b[+$ ]$c, d[$ indeed.

Example 4.2. Define the numbers $\alpha, \beta, \gamma, \delta$ by (5). Let

$$
\left.x_{0}:=4.8+0.1 \sqrt{2}=4.9414 \cdots \in\right] \alpha+\gamma, \beta+\delta[.
$$

We show that there exist constants $\left.x^{*} \in\right] \alpha, \beta\left[\right.$ and $\left.y^{*} \in\right] \gamma, \delta[$ such that $x_{0}=x^{*}+y^{*}$. Preserving the notations of Proporition 4.1. we obtain that

$$
A_{1}=0.8535 \ldots, \quad A_{2}=1.3535 \ldots, \quad A_{3}=1.0798 \ldots, \quad A_{4}=0.7870 \ldots
$$




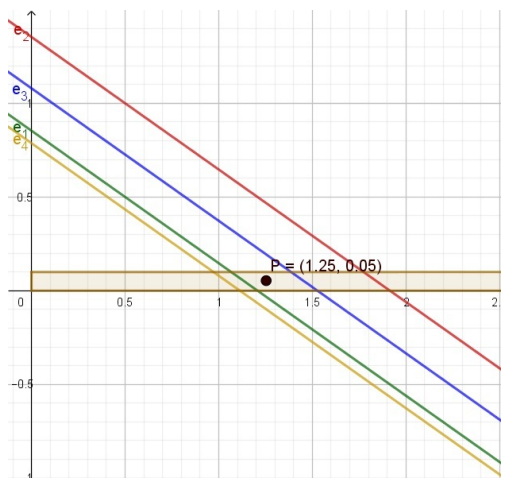

Figure 1: Sums of intervals

Consider the Figure $1{ }^{*}$ :

It is easy to see that the choice of $P:=(1.25,0.05)$ is convenient. Thus we obtain that

$$
\begin{aligned}
& \left.x^{*}=1.25+0.05 \sqrt{2} \in\right] \alpha, \beta[, \\
& \left.y^{*}=3.55+0.05 \sqrt{2} \in\right] \gamma, \delta[
\end{aligned}
$$

with $x^{*}+y^{*}=x_{0}$.

Proposition 4.3. Let $a, b, c, d \in K_{1}$ such that $a<b$ and $c<d$. Then

$$
] a, b[\cdot] c, d[=] a c, b d[\text {. }
$$

Proof. The inclusion $] a, b[\cdot] c, d[\subseteq] a c, b d[$ is clear. For the converse inclusion let

$$
\left.x_{0}:=u+v \sqrt{2} \in\right] a c, b d[.
$$

We find elements $x^{*}, y^{*} \in K_{1}$ such that

$$
\left.x^{*}=x+y \sqrt{2} \in\right] a, b\left[, \quad y^{*}=\frac{x_{0}}{x^{*}} \in\right] c, d[.
$$

Now we list the necessary and sufficient conditions for $\left.x^{*} \in\right] a, b\left[\right.$ and $\left.y^{*} \in\right] c, d[$.

1. $a<x^{*}$ which is equivalent to the inequality $e_{1}(x)<y$ where

$$
e_{1}(x)=-\frac{1}{\sqrt{2}} x+A_{1} \quad \text { and } \quad A_{1}=\frac{a}{\sqrt{2}}
$$

* The Figure 1 was made with the program GeoGebra 
2. $x^{*}<b$ which is equivalent to the inequality $y<e_{2}(x)$ where

$$
e_{2}(x)=-\frac{1}{\sqrt{2}} x+A_{2} \quad \text { and } \quad A_{2}=\frac{b}{\sqrt{2}}
$$

3. $c<y^{*}$ which is equivalent to the inequality $y<e_{3}(x)$ where

$$
e_{3}(x)=-\frac{1}{\sqrt{2}} x+A_{3} \quad \text { and } \quad A_{3}=\frac{x_{0}}{\sqrt{2} c} ;
$$

4. $y^{*}<d$ which is equivalent to the inequality $e_{4}(x)<y$ where

$$
e_{4}(x)=-\frac{1}{\sqrt{2}} x+A_{4} \quad \text { and } \quad A_{4}=\frac{x_{0}}{\sqrt{2} d} .
$$

Now we list the necessary and sufficient conditions for $x^{*} \in K_{1}$ and $y^{*} \in K_{1}$ :

Since

$$
\frac{4 x-2 v y}{x^{2}-2 y^{2}}>0, \quad \text { and } \quad \frac{-u y+v x}{x^{2}-2 y^{2}}>0,
$$

thus (6) is fulfilled if and only if, $y<\mu x$ or $\nu x<y$ where the constants $\mu$, $\nu \in \mathbb{R}_{+}$are defined by

$$
\mu:=\min \left\{\frac{1}{\sqrt{2}}, \frac{2}{v}, \frac{v}{u}\right\}, \quad \nu:=\max \left\{\frac{1}{\sqrt{2}}, \frac{2}{v}, \frac{v}{u}\right\} .
$$

Define the domains $D_{1}, D_{2}$ by

$$
\begin{aligned}
& D_{1}:=\left\{(x, y) \in \mathbb{Q}_{+}^{2} \mid e_{1}(x)<y, y<e_{2}(x), y<e_{3}(x), e_{4}(x)<y\right\}, \\
& D_{2}:=\left\{(x, y) \in \mathbb{Q}_{+}^{2} \mid y<\mu x \text { or } \nu x<y\right\} .
\end{aligned}
$$

Since $\mathbb{Q}^{2}$ is dense in $\mathbb{R}^{2}$ thus we obtain that $D_{1} \cap D_{2} \neq \emptyset$. Choose a point $P=P(x, y) \in\left(D_{1} \cap D_{2}\right)$ arbitrarily and define the numbers $x^{*}$ and $y^{*}$ by

$$
x^{*}:=x+\sqrt{2} y \quad \text { and } \quad y^{*}:=\frac{x_{0}}{x^{*}} .
$$

Thus $\left.x^{*} \in\right] a, b\left[\right.$ and $\left.y^{*} \in\right] c, d[$ whence we obtain that $] a c, b d[\subseteq] a, c[\cdot] b, c[$ indeed.

Example 4.4. Define the numbers $\alpha, \beta, \gamma, \delta$ by (5). Let

$$
\left.y_{0}:=4+0.1 \sqrt{2}=4.1414 \cdots \in\right] \alpha \gamma, \beta \delta[.
$$

We show that there exist constants $\left.x^{*} \in\right] \alpha, \beta\left[\right.$ and $\left.y^{*} \in\right] \gamma, \delta[$ such that $y_{0}=x^{*} y^{*}$. Preserving the notations of Proporition 4.3. we obtain that

$$
A_{1}=0.8535 \ldots, \quad A_{2}=1.3535 \ldots, \quad A_{3}=0.8577 \ldots, \quad A_{4}=0.7649 \ldots,
$$

moreover, $\mu=0.025, \nu=20$. 
Consider the following figures ${ }^{\dagger}$ :

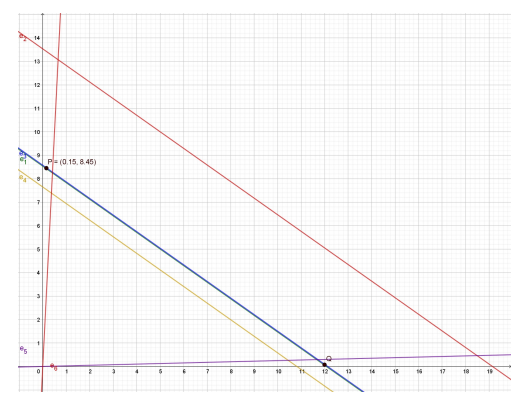

Figure 2: Products of intervals

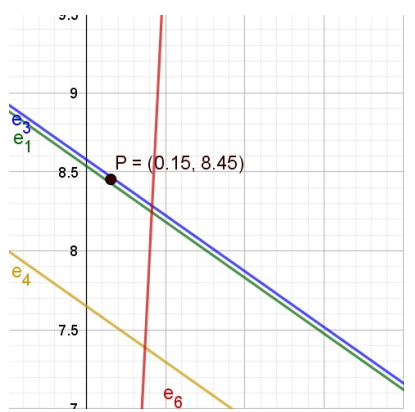

Figure 3: Magnification of Figure 2

The Figure 2 and Figure 3 were taken at ten times magnification for better illustration. The lines $e_{1}, e_{2}, e_{3}, e_{4}$ are defined by

$$
\begin{array}{ll}
e_{1}=-0.71 x+8.54, & e_{3}=-0.71 x+8.58, \\
e_{2}=-0.71 x+13.54, & e_{4}=-0.71 x+7.65 .
\end{array}
$$

The lines $e_{5}, e_{6}$ are determined by (7) and defined by

$$
e_{5}=0.03 x, \quad e_{6}=20 x .
$$

It is easy to see that the point $P:=(0.015,0.845)$ is convenient. (The point $Q$ is also convenient.) Thus we obtain that

$$
\begin{aligned}
& \left.x^{*}=0.015+0.845 \sqrt{2}=1.21 \cdots \in\right] \alpha, \beta[, \\
& \left.y^{*}=\frac{0.109+3.3785 \sqrt{2}}{1.427825}=3.4226 \cdots \in\right] \gamma, \delta[
\end{aligned}
$$

with $x^{*} y^{*}=y_{0}$.

Theorem 4.5. Let $a, b, c d \in K_{n}$ such that $a<b$ and $c<d$. Then

$$
\begin{aligned}
] a, b[+] c, d[ & =] a+c, b+d[, \\
] a, b[\cdot] c, d[ & =] a c, b d[.
\end{aligned}
$$

Proof. The proof is easy by induction using Proposition 4.1. and Proposition 4.3.

\footnotetext{
${ }^{\dagger}$ The Figure 2 and Figure 3 were made with the program GeoGebra
} 
Summarising the above Propositions and Theorem concerning the structure $K_{1}\left(\right.$ or $K_{n}$ ) we can infer, that:

$K_{1}$ has no property (2) of Definition 2.1. For example $\alpha<\beta$ but $\beta-\alpha=$ $0.5 \sqrt{2} \notin K_{1}$.

Equality (1) is fulfilled but equality (3) is not. For example let $x_{0}=4.8+$ $0.1 \sqrt{2}=4.9414 \ldots$ Then $\left.x_{0} \in\right] \alpha+\gamma, \alpha+\delta\left[\right.$ but $x_{0}-\alpha=4.3-0.4 \sqrt{2} \notin K_{1}$

Equality (2) is fulfilled but equality (4) is not. For example let $y_{0}=$ $4+0.1 \sqrt{2}=4.1412$ then $\left.y_{0} \in\right] \alpha \gamma, \alpha \delta\left[\right.$ but $\frac{y_{0}}{\alpha}=-7.6+7.8 \sqrt{2} \notin K_{1}$.

\section{$5 \quad$ Results and Problems}

The example of $K_{n}$ and $\mathbb{Q}_{n}$ motivates the problems bellow.

Problem 5.1. How to characterise the semirings that satisfy equalities (1) and (2) but do not satisfy equalities (3) and (4)?

The first author of this article has proven the following Theorem in [6]:

Theorem Let $\mathbb{G}(+, \leqslant)$ be an Archimedean ordered dense Abelian group, $Y(+)$ be a group, $x_{0}, y_{0} \in \mathbb{G}, \varepsilon \in \mathbb{G}_{+}$, moreover, let

$$
\begin{aligned}
& f:] x_{0}+y_{0}-2 \varepsilon, x_{0}+y_{0}+2 \varepsilon[\rightarrow Y, \\
& g:] x_{0}-\varepsilon, x_{0}+\varepsilon[\rightarrow Y, \\
& h:] y_{0}-\varepsilon, y_{0}+\varepsilon[\rightarrow Y
\end{aligned}
$$

be functions such that

$$
f(x+y)=g(x)+h(y) \quad(x \in] x_{0}-\varepsilon, x_{0}+\varepsilon[, y \in] y_{0}-\varepsilon, y_{0}+\varepsilon[),
$$

then there exists an additive function $a: \mathbb{G} \rightarrow Y$ and exist constants $c, d \in Y$ such that

$$
\begin{aligned}
f(w) & =a(w)+c+d \quad(w \in] x_{0}+y_{0}-2 \varepsilon, x_{0}+y_{0}+2 \varepsilon[), \\
g(u) & =a(u)+c \quad(u \in] x_{0}-\varepsilon, x_{0}+\varepsilon[), \\
h(v) & =a(v)+d \quad(w \in] y_{0}-\varepsilon, y_{0}+\varepsilon[) .
\end{aligned}
$$

The proof is based on the equalities (1) and (3).

Problem 5.2. Can a theorem analogous to the above one be proved in that case when the role of group $\mathbb{G}$ is taken over by a semigroup $\mathbb{S}$ in which equality (1) is satisfied but equality (3) is not?

Conjecture 5.3. If $\mathbb{S}=\mathbb{S}(+, \leqslant)$ is a dense ordered semigroup, then (1) is satified, that is

$$
] a, b[+] a, d[=] a+c, b+d[
$$

for all $a, b, c, d \in \mathbb{S}$ such that $a<b, c<d$. 


\section{References}

[1] M. El Bachraoui, Primes in the interval [2n; 3n], Int. J. Contemp. Math. Sciences, 1(13) (2006), 617-621.

[2] A.M. Bruckner, J.B. Bruckner, B.S. Thomson, Elementary Real Analysis, Prentice-Hall (2001)

[3] P. Erdös, Beweis eines satzes von tschebyschef. Acta Litt. Univ. Sci., Szeged, Sect. Math., 5 (1932), 194-198.

[4] L. Fuchs, Partially Ordered Algebraic Systems, Dover Publications, Inc. Minesota, New York. (1963)

[5] P. Erdei, T. Glavosits On the functional equation $f(x+y)=g(x y),($ In preparation)

[6] T. Glavosits, Short remark to the Rimán's Theorem, (In preparation)

[7] T. Glavosits, Zs. Karácsony, On the restricted Pexider additive functional equations on rectangulars of $\mathbb{Z}^{2}$, (In preparation)

[8] T. Glavosits, Zs. Karácsony, Sums and products of intervals in ordered semigroups and fields, (accepted in Acta Univ. Sap., Math. (2021)

[9] T. Glavosits, Á. Száz, On the existence of nonnegativity domains of subsets of groups. Demonstratio Math. 37 (2004), 505-516.

[10] F. W. Levi, Arithmetische Gesetze im Gebiete diskreter Gruppen, Rend. Circ. Mat. Palermo 35 (1913), 225-236.

[11] P. Lorenzen, Abstrakte Begründung der multiplikativen Idelatheorie, Math. Z. 45 (1939), 533-553.

[12] R. E. Moore, Automatic error analysis in digital computation. Technical Report LMSD-48421 Lockheed Missiles and Space Co, Palo Alto, CA., (1959)

[13] H. Simbireva, On the theory of partially ordered groups, Mat. Sb.20 (1947), 145-178. (in Russian)

Tamás GLAVOSITS,

Department of Applied Mathematics,

University of Miskolc,

Miskolc-Egyetemváros, Hungary, H-3515.

Email: matgt@uni-miskolc.hu

Zsolt KARÁCSONY,

Department of Applied Mathematics,

University of Miskolc,

Miskolc-Egyetemváros, Hungary, H-3515.

Email: matkzs@uni-miskolc.hu 